Computing 8, 32-39 (1971)

(C) by Springer-Verlag 1971

\title{
Verfahren zur Berechnung des Spektralradius nichtnegativer irreduzibler Matrizen
}

\author{
Von \\ L. Elsner, Erlangen \\ (Eingegangen am 26. Juni 1970) \\ Zusammenfassung - Summary
}

Verfahren zur Berechnung des Spektralradius nichtnegativer irreduzibler Matrizen. Es werden mehrere Iterationsverfahren zur Bestimmung des Spektralradius $\varrho$ einer nichtnegativen Matrix $A$ angegeben. Thre Konvergenz wird unter der Voraussetzung bewiesen, da $B A$ irreduzibel ist. Die Verfahren sind, im Gegensatz zur Potenzmethode, unempfindlich gegen das Vorhandensein mehrerer Eigenwerte vom Betrage @.

Methods for Computing the Spectral Radius of Nonnegative Irreducible Matrices. Several iterative methods for determining the spectral radius $\varrho$ of a nonnegative matrix $A$ are given. Convergence is proved under the assumption of irreducibility. Contrary to the power method, these methods are insensitive to the presence of other eigenvalues with modulus $\varrho$.

\section{Einleitung}

Es sei $\Im$ die Menge der nichtnegativen irreduziblen $N \times N$-Matrizen, $A \in \Im$ und $\omega=\varrho(A)$ der Spektralradius von $A$. Dann gibt es einen positiven Vektor $y>0$ mit $A y=\omega y$. Die Bestimmung von $y$ ist bekanntlich äquivalent mit der Bestimmung einer Diagonalmatrix $Y$ mit positiven Diagonalelementen, so daß $Y^{-1} A Y$ konstante Zeilensummen hat. Bei den meisten numerischen Verfahren wird $Y$ als unendliches Produkt

$$
Y=D_{1} D_{2} D_{3} \ldots
$$

von Diagonalmatrizen der Gestalt

$$
D_{i}=\operatorname{diag}\left(1, \ldots, 1, d_{i}, 1, \ldots, 1\right)
$$

aufgebant. Sei $A_{i}=D_{1} D_{2} \ldots D_{i}$ und $A_{i}=\Delta_{i}{ }^{-1} A A_{i}$. In dieser Darstellung erseheinen die Verfahren am einfachsten, bei denen die $D_{i}$ nur aus $A_{i-1}$ berechnet werden. Solche Verfahren kann man etwa Einschrittverfahren nennen, im Gegensatz zu $k$-Schrittverfahren, wo $D_{i}$ eine Funktion von $A_{i-1}, \ldots, A_{i-k}$ ist. In dieser Terminologie ist die Potenzmethode ein $2 N$-Schrittverfahren. Die Verfahren in [1] und [3] stellen $N$-Schrittverfahren dar. 
Einschrittverfahren wurden in [2] behandelt. Der dort angegebene Algorithmus konvergiert allerdings nicht für alle $A \in \Im$. In der vorliegenden Arbeit geben wir Einschrittverfahren an, die für alle nichtnegativen irreduziblen Matrizen konvergieren.

Nach einigen Vorbereitungen wird das Hauptergebnis in Satz 3 formuliert. Er gibt ziemlich allgemeine Kriterien für die Auswahl der $d_{i}$ an, die die Konvergenz für alle $A \in \Im$ garantieren. Anhand dieser Ergebnisse werden im vierten Abschnitt einige spezielle Algorithmen angegeben. Insbesondere wird gezeigt, wie das Verfahren in [2] modifiziert werden

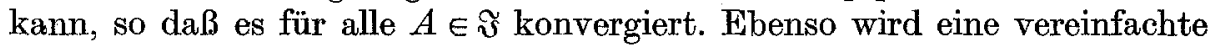
Variante von [3] angegeben. Schließlich wird über (nicht sehr umfangreiche) numerische Erfahrungen berichtet.

\section{Hilfsbetrachtungen}

Wir verwenden im folgenden die Bezeichnungsweise $x>0$ bzw. $x \geqslant 0$ für positive bzw. nichtnegative Vektoren. Analoges gilt für Matrizen. Ist $x$ ein Vektor, so sei $\|x\|=\max _{i}\left|x_{i}\right|$.

Es sei $m=\min \left\{a_{i k} \mid a_{i k}>0, i \neq k\right\}$.

Satz 1. Sei $x>0$ ein gegebener Veltor, $\varepsilon>0$ und

Sei

$$
A x \leqslant \omega(1+\varepsilon) x .
$$

Dann gilt

$$
\gamma(\varepsilon)=1+\frac{(1+\varepsilon)^{N-1}-1}{m^{N-1} \min _{i, k}\left(\frac{y_{i}}{y_{k}}\right)} \omega^{N-1} .
$$

und

$$
\min _{i} \frac{x_{i}}{y_{i}} \leqslant \frac{x_{k}}{y_{k}} \leqslant \gamma(\varepsilon) \min _{i} \frac{x_{i}}{y_{i}} \quad k=1, \ldots, N
$$

$$
A x \geqslant \frac{\omega}{\gamma(\varepsilon)} x .
$$

Beweis. Wir betrachten zunächst den Fall $\omega=1, y=(1, \ldots, 1)$, also $A$ stochastisch.

Sei $x_{j}=\min _{i} x_{i}$. Zu gegebenem $k \neq j$ existiert wegen der Irreduzibilität von $A$ eine Zahl $s, 1 \leqslant s \leqslant N-1$ mit $\left(A^{s}\right)_{j, k}=a_{j k}^{(s)}>0$. Wegen $A^{s} x \leqslant(1+\varepsilon)^{s} x, A^{s} y=y$ gilr

$$
\sum_{n} a_{j n}^{(s)}\left(\frac{x_{n}}{x_{j}}-1\right) \leqslant(1+\varepsilon)^{s}-1
$$

Da $x_{j}$ minimal ist, sind alle Summanden der linken Seite nichtnegativ, also

$$
\frac{x_{k}}{x_{j}}-1 \leqslant\left((1+\varepsilon)^{s}-1\right) / a_{j k}^{(s)}
$$

und daher 


$$
x_{j} \leqslant x_{k} \leqslant\left(1+\frac{(\mathbf{1}+\varepsilon)^{s}-\mathbf{1}}{a_{j k}^{(s)}}\right) x_{j} .
$$

Wegen $a_{j k}^{(s)} \geqslant m^{s} \geqslant m^{N-1}$ gilt nun (2). Es folgt $x_{k} / x_{i} \geqslant \gamma^{-1}$ für beliebige $i, k$ und daher

also $(3)$.

$$
\sum_{k} a_{i k} \frac{x_{k}}{x_{i}} \geqslant \gamma^{-1} \sum_{k} a_{i k}=\gamma^{-1}
$$

Der allgemeine Fall wird nun auf diesen Fall zurückgeführt. Ist $Y$ die Diagonalmatrix mit den Diagonalelementen $y_{i}$, so ist $B=\omega^{-1} Y^{-1} A Y$ stochastisch und $B Y^{-1} x \leqslant(1+\varepsilon) Y^{-1} x$. Für $B$ und $Y^{-1} x$ ist bereits alles bewiesen. Durch Rücktransformation folgen die Aussagen auch für den allgemeinen Fall.

Bemerkung. Ist $A>0$, so ist stets $s=1$ möglich. Daher gilt dann (2) und (3) schon mit

$$
\gamma(\varepsilon)=1+\frac{\varepsilon \omega}{m} \max _{i, k} \frac{y_{i}}{y_{k}} .
$$

Es sei im folgenden $y$ normiert, $\|y\|=1$.

$x^{n} \quad(n=1,2, \ldots)$ sei eine Folge positiver Vektoren, $\left\|x^{n}\right\|=1$, $x^{n}=\left(x_{1}^{n}, \quad x_{2}^{n}, \ldots, x_{N}^{n}\right), \quad R_{i}^{n}=\sum_{k} a_{i k} x_{k}^{n} / x_{1}^{n} \quad i=1, \ldots, N \quad n=1,2, \ldots$ $R_{n}=\max _{i} R_{i}^{n}$.

Satz 2. Es sei $\lim R_{n}=\omega$. Dann gilt

$$
\begin{aligned}
& \lim R_{i}^{n}=\omega \quad i=1, \ldots, N, \\
& \lim x^{n}=y .
\end{aligned}
$$

Beweis. Es ist $A x^{n} \leqslant R_{n} x^{n}=\omega\left(1+\varepsilon_{n}\right) x^{n}$ und offenbar lim $\varepsilon_{n}=0$. Daher strebt $\gamma\left(\varepsilon_{n}\right)$ gegen 1. (3) zeigt nun die Behauptung (4).

Ist $x$ ein Häufungspunkt von $\left\{x^{n}\right\}$, so zeigt (2), daß $x$ ein Vielfaches von $y$ ist. Wegen der Normierung ist $x=y .\left\{x^{n}\right\}$ besitzt also nur einen Häufungspunkt, nämlich $y$. Daher konvergiert $\left\{x^{n}\right\}$ gegen $y$. Q. e. d.

Bemerkung. Satz 2 findet sich im wesentlichen bereits in [2]. Die Herleitung über Satz 1, der quantitative Aussagen enthält, ist neu.

\section{Eine Klasse von Verfahren}

Wir konstruieren rekursiv eine Vektorfolge $x^{n}$ : Sei $x^{1}=(1, \ldots, 1)$. Es sei $v=v(n), \mu=\mu(n)$ ausgewählt, so daß

$$
R_{\nu}^{n} \leqslant R_{i}^{n} \leqslant R_{\mu}^{n} \quad i=1, \ldots, N
$$

gilt. Mit einer noch zu bestimmenden Größe $d=d_{n}$ sei $\widetilde{x}=\left(\widetilde{x}_{k}\right)$ mit

$$
\widetilde{x}_{k}=\left\{\begin{array}{ll}
x_{k}^{n} & k \neq \nu \\
d x_{v}^{n} & k=\nu
\end{array} \quad x^{n+1}=\widetilde{x} /\|\widetilde{x}\| .\right.
$$


Dann können wir den folgenden Konvergenzsatz beweisen.

Satz 3. $d_{n}$ werde so gewählt, daß folgende Aussagen gelten:

(a) $0<d_{n}<1$, falls $R_{v}^{n} \neq R_{\mu}^{n}$.

(b) Existiert eine Teilfolge $n(i)$ mit $d_{n(i)} \rightarrow 1$, so folgt $\lim R_{n}=\omega$.

(c) Es existiert eine Zahl $\alpha, 0<\alpha<1, \alpha$ unabhängig von $n$ mit $R_{x}^{n+1} \leqslant \propto R_{\mu}^{n}+(1-\alpha) R_{v}^{n}$.

Dann ist $\lim R_{n}=\omega$ und es sind (4) und (5) erfüllt.

Beweis. Wegen Satz 2 genügt es, $\lim R_{n}=\omega$ zu zeigen.

Allgemein gilt

$$
\begin{gathered}
R_{i}^{n+1}=R_{i}^{n}+\left(d_{n}-1\right) a_{i v} \frac{x_{v}^{n}}{x_{i}^{n}}, \quad i \neq v, \\
R_{v}^{n+1}=a_{v v}+\frac{R_{v}^{n}-a_{v v}}{d_{n}} .
\end{gathered}
$$

Aus (a) und (c) folgt nun sofort

$$
R_{n} \geqslant R_{n+1} \geqslant \ldots
$$

Die $R_{i}$ sind also monoton fallend und nach unten durch $\omega$ beschränkt.

Es sei $I=\{p \in\{1, \ldots, N\}, p=\nu(n)$ nur für endlich viele $\mathrm{n}\}$. Wir unterscheiden zwei Fälle.

Fall 1. $I \neq \emptyset$. $\mathrm{Da} I \neq\{1, \ldots, N\}$ ist, gibt es wegen der Irreduzibilität von $A$ ein $k \in I, l \notin I$ mit $a_{k l}>0$.

Wegen (8) und (2) ist für alle $n$ die Größe $a_{k l}^{n}=a_{k l} x_{l}^{n} \mid x_{k}^{n}$ nach unten beschränkt. Es sei für alle $n \geqslant N_{0}: \nu(n) \neq k$. Dann ist wegen (6) für $n \geqslant N_{0}$

$$
R_{k}^{n+1}=R_{k}^{n}+a_{i v}^{n}\left(d_{n}-1\right) .
$$

Ist $1-d_{n} \geqslant \varepsilon>0$ für alle $n$, so folgt weiter

$$
R_{k}^{n+s} \leqslant R_{k}^{n}-\varepsilon \sum_{r=n}^{n+s-1} a_{k v(r)}^{r}=R_{k}^{n}-\varepsilon w_{s} .
$$

Da immer wieder $v(r)=l$ ist, gilt $\lim w_{s}=\infty$, ein Widerspruch gegen $R_{k}^{n+s}>0$. Es gibt daher eine Teilfolge $n(i)$ mit $d_{n(i)} \rightarrow 1$. Mit (b) folgt die Behauptung.

Fall 2. $I=\emptyset$. Sei $p$ ein Index, so daß für abzählbar viele $n$ gilt $\mu(n)=p$. Durch Induktion läßt sich die Existenz von Zahlenfolgen $m(i), n(i)$ zeigen mit

$$
\begin{aligned}
& m(i)<n(i)<m(i+1), \\
& \nu(m(i))=\mu(n(i))=p, \\
& m(i)<n<n(i) \Rightarrow v(n) \neq p .
\end{aligned}
$$


Dann gilt offenbar

Nach (c) folgt

$$
R_{p}^{m(i)+1} \geqslant R_{p}^{n(i)}=R_{n(i)}
$$

$$
\begin{aligned}
0 & \leqslant R_{n(i)}-\omega \leqslant R_{p}^{m(i)+1}-\omega \leqslant \alpha R_{\mu(m(i))}^{m(i)}+(1-\alpha) R_{p}^{m(i)}-\omega \\
& \leqslant \alpha\left(R_{\mu(m(i))}^{m(i)}-\omega\right)=\alpha\left(R_{m(i)}-\omega\right) .
\end{aligned}
$$

Zusammen mit (8) folgt daraus die Behauptung. Q. e. d.

\section{Spezielle Verfahren}

Satz 3 legt das folgende Verfahren nahe:

Verfahren 1. $\mathrm{Zu}$ vorgegebenem $0<\alpha<1$ wähle $d_{n}$ so, daß

ist. Das führt auf

$$
R_{v}^{n+1}=\alpha R_{\mu}^{n}+(1-\alpha) R_{y}^{n}
$$

$$
d_{n}=\frac{R_{v}^{n}-a_{v v}}{\alpha R_{\mu}^{n}+(1-\alpha) R_{v}^{n}-a_{v v}}=\frac{R_{v}^{n}-a_{v v}}{R_{v}^{n}-a_{v v}+\alpha\left(R_{\mu}^{n}-R_{v}^{n}\right)} .
$$

Damit ist offenbar (a) und (c) von Satz 3 erfüllt. Aus $d_{n(i)} \rightarrow 1$ folgt $R_{\mu}^{n(i)}-R_{v}^{n(i)} \rightarrow 0$, und wegen $R_{v}^{n} \leqslant \omega \leqslant R_{\mu}^{n}$ gilt $\lim R_{n(i)}=\omega$, also wegen (8) auch $\lim R_{n}=\omega$. Mithin ist (b) erfüllt. Verfahren 1 konvergiert daher für alle nichtnegativen irreduziblen $A$.

Etwas komplizierter ist das folgende Verfahren, das im wesentlichen eine vereinfachte Version des in [3] vorgeschlagenen Algorithmus ist. Wie in [3] sei

$$
\begin{aligned}
l_{i}^{n}(t) & =R_{i}^{n}+a_{i v(n)}^{n}(t-1) \quad i \neq v(n), \\
h_{v}^{n}(t) & =a_{v v}+\left(R_{v}^{n}-a_{v v}\right) / l .
\end{aligned}
$$

Es sei $\xi$ eine Lösung von $l_{\mu}^{n}(\xi)=h_{p}^{n}(\xi)$. Dann genügt $\xi$ der Gleichung

$$
g(\xi) \equiv a_{u v}^{n}\left(\xi^{2}-\xi\right)+\left(R_{\mu}^{n}-a_{v v}\right) \xi+\left(a_{v v}-R_{v}^{n}\right)=0 .
$$
Wegen $g(0)<0, g(1)>0, g\left(\xi_{0}^{n}\right) \leqslant 0$ für $\xi_{0}^{n}=\frac{a_{v v}-R_{v}^{n}}{a_{v v}-R_{\mu}^{n}}$ gibt es genau
eine Lösung $\xi$ in

für die sogar

$$
0<\xi<1,
$$

$$
\frac{R_{p}^{n}-a_{v v}}{R_{\mu}^{n}-a_{v v}} \leqslant \xi<1
$$

gilt. Der explizite Wert von $\xi$ ist

$$
\xi=\xi^{n}=\left\{\begin{array}{c}
\frac{R_{\nu}^{n}-a_{v v}}{R_{\mu}^{n}-a_{v v}} \cdot\left(a_{\mu \nu}=0\right) \\
\frac{a_{\mu \nu}^{n}+a_{v v}-R_{\mu}^{n}+\left(\left(R_{\mu}^{n}-a_{\mu \nu}^{n}-a_{v v}\right)^{2}+4 a_{\mu \nu}^{n}\left(R_{v}^{n}-a_{v v}\right)\right)^{\frac{1}{2}}}{2 a_{\mu \nu}^{n}} \cdot\left(a_{\mu \nu}>0\right)
\end{array}\right.
$$


Verfahren 2. Sei $0<\alpha<1, \beta=\alpha^{-1}$ und

$$
d_{n}=\alpha\left(\xi_{n}-1\right)+1=\alpha \xi_{n}+(1-\alpha) \cdot 1 .
$$

Wegen $0<\xi_{n}<1$ gilt $1-\alpha<d_{n}<1$.

Wir verifizieren die Voraussetzungen von Satz 3.

(a) ist trivial, (b) rechnet man unmittelbar nach.

(c): Für reelle Zahlen $0<\eta \leqslant \zeta$ gilt, wie man leicht beweist,

$$
\frac{\eta}{\alpha \zeta+(1-\alpha) \eta} \leqslant \frac{(1-\alpha) \zeta+\alpha \eta}{\zeta} \text {. }
$$

Aus (14) folgt $1+\beta\left(d_{n}-1\right) \geqslant \frac{R_{v}^{n}-a_{v v}}{R_{\mu}^{n}-a_{v v}}$. Daher

$$
d_{n} \geqslant \frac{(1-\alpha)\left(R_{\mu}^{n}-a_{v v}\right)+\alpha\left(R_{\nu}^{n}-a_{v v}\right)}{R_{\mu}^{n}-a_{v v}}
$$

und wegen (16)

$$
\geqslant \frac{R_{v}^{n}-a_{\nu y}}{\alpha\left(R_{\mu}^{n}-a_{\nu v}\right)+(1-\alpha)\left(R_{v}^{n}-\alpha_{\nu v}\right)} .
$$

Aus (10) und (7) folgt dann

$$
R_{v}^{n+1} \leqslant \alpha R_{\mu}^{n}+(1-\alpha) R_{v}^{n}, \quad \text { Q. e. d. }
$$

Bemerkung. Unter der in der Praxis wohl stets erfüllten Voraussetzung, daß für alle $n$ die Relation $R_{i}^{n}=R_{n}$ nur für ein $i$ gilt, fällt für $\alpha=1 / 2$ Verfahren 2 mit dem in [3] angegebenen Algorithmus zusammen. Allerdings ist unser Verfahren einfacher zu programmieren.

In [2] wird der Fall $\alpha=1$ betrachtet. Dann ist

$$
R_{v}^{n+1}=R_{\mu}^{n+1} \text {. }
$$

(a) und (b) sind erfüllt. Ist $a_{\mu v}>0$, so folgt aus (18), (6) und (7)

$$
R_{v}^{n+1}-R_{\nu}^{n}=\frac{R_{v}^{n}-a_{v v}}{d_{n} a_{\mu \nu}^{n}}\left(R_{\mu}^{n}-R_{\nu}^{n+1}\right) .
$$

Wegen (17) für $\alpha=1$ folgt

$$
\leqslant \frac{R_{\mu}^{n}-a_{v v}}{a_{\mu \nu}^{n}}\left(R_{\mu}^{n}-R_{v}^{n+1}\right) \leqslant K\left(R_{\mu}^{n}-R_{v}^{n+1}\right),
$$

wobei $K$ unabhängig von $n$ wählbar ist. Es folgt

$$
R_{v}^{n+1} \leqslant \frac{K}{1+K} R_{\mu}^{n}+\frac{1}{1+K} R_{v}^{n} .
$$

Für $a_{\mu \nu}=0$ ist wegen $R_{v}^{n+1}=R_{\mu}^{n+1}=R_{\mu}^{n}$ eine solche Ungleichung nicht mehr möglich. Wie in [2] gezeigt wird, konvergiert dieses Verfahren auch nicht für alle irreduziblen $A$. Es ist nun klar, wie dieser Algorithmus abzuändern ist, damit Konvergenz eintritt: Im Fall $a_{\mu v}=0 \mathrm{mu}$ die 
Ungleichung in (c) erzwungen werden. Das kann dadurch geschehen, indem man dann $d_{n}$ wie in Verfahren 1 oder 2 wählt. Wir notieren nur die erste Möglichkeit.

Verfahren 3. Wähle mit einem $0<\alpha<1$

$$
d_{n}=\left\{\begin{array}{l}
\xi_{n} \quad \text { falls } a_{\mu v}>0 \\
\frac{R_{v}^{n}-a_{v v}}{\alpha R_{\mu}^{n}+(1-\alpha) R_{v}^{n}-a_{v v}}
\end{array} \quad \text { falls } a_{\mu v}=0 .\right.
$$

Die Konvergenz ist nach Satz 3 gesichert.

Bemerkung. Ist die folgende Bedingung erfüllt

$$
\exists p \text { mit } a_{i p}>0 \text { für alle } i=1, \ldots, N, i \neq p,
$$

so konvergiert das Verfahren 2 auch für $\alpha=1$. Da die Bedingung (c) von Satz 3 nicht erfüllt ist, muß der Konvergenzbeweis im Fall $2(I=\emptyset)$ modifiziert werden. Das geschieht wie in [2]. Da $p \notin I$, existiert eine Folge $k(i)$ mit $v(k(i))=p$. Daher ist für ein $j \neq p$

Daher

$$
\begin{aligned}
R_{k(i)+1} & =R_{j}^{k(i)+1}=R_{j}^{k(i)}+a_{j p}^{k(i)}\left(d_{k(i)}-1\right) \\
& \leqslant R_{k(i)}+a_{j p}^{k(i)}\left(d_{k(i)}-1\right) .
\end{aligned}
$$

$$
0<1-d_{k(i)} \leqslant\left(R_{k(i)}-R_{k(i)+1}\right) / a_{j p}^{k(i)} .
$$

Da die $R_{n}$ konvergieren, geht die rechte Seite gegen Null, d. h. $d_{k(i)} \rightarrow 1$ und wegen (b) gilt $R_{n} \rightarrow \omega$. Q. e. d.

Diese hinreichende Bedingung ist schwächer als die in [2] angegebene.

\section{Beispiele}

Es wurden etliche numerische Beispiele durchgerechnet, um einen Vergleich zwischen den Verfahren zu ermöglichen und den Einfluß des Parameters $\alpha \mathrm{zu}$ studieren. Dabei ergab sich folgendes Bild, wenn man die Anzahl der Irerationsschritte zur Erreichung einer gewissen Genauigkeit $R_{\mu}^{n}-R_{v}^{n}$ zugrundelegt.

Bei vollbesetzter Matrix war das Verfahren 3 (in diesem Fall identisch mit dem Verfahren von [2]) eindeutig überlegen. Beim Auftreten von Nullen verschob sich das Bild: Hier erwiesen sich alle drei Verfahren als etwa gleich gut. Zudem zeigte sich, daß es dann jeweils am günstigsten war, $\alpha$ im Bereich 0.5-0.7 zu wählen. Legt man die Anzahl der Reehenschritte zugrunde, so schneidet Verfahren 1 wegen seiner Einfachheit etwas günstiger ab. Alle Verfahren erwiesen sich als unempfindlich gegen das Vorhandensein von Eigenwerten mit Beträgen nahe bei $\varrho(A)$, wie auch in [2] beobachtet wurde.

Als Beispiel für schwachbesetzte Matrizen, deren Eigenwerte zusätzlich alle den gleichen Betrag haben, führen wir 


$$
A=\left(\begin{array}{llll}
0 & 1 & 0 & 0 \\
0 & 0 & 0.5 & 0 \\
0 & 0 & 0 & 0.25 \\
1 & 0 & 0 & 0
\end{array}\right)
$$

an. In der folgenden Tabelle sind die Anzahl der Schritte angegeben, um $R_{\mu}-R_{p} \leqslant 10^{-4} \mathrm{zu}$ erreichen.

\begin{tabular}{c|c|c|c}
\hline$\alpha$ & Verfahren 1 & Verfahren 2 & Verfahren 3 \\
\hline 0.9 & 82 & 100 & 129 \\
0.7 & 28 & 28 & 34 \\
0.5 & 16 & 30 & 20 \\
0.3 & 29 & 49 & 20 \\
0.1 & 115 & 195 & 76
\end{tabular}

Das Verfahren 2 mit $\alpha=1$ ist hier zufällig nach 6 Schritten exakt am Ziel.

\section{Literatur}

[1] Brauer, A.: On the Characteristic Roots of Non-negative Matrices, in: Recent Advances in Matrix Theory (ScHNEIDER H., ed.). Madison: University of Wisconsin Press. 1964.

[2] HaLI, C. A., and T. A. Porsohing: Computing the maximal eigenvalue and eigenvector of a positive matrix. SIAM J. Num. Anal. 5, 269-274 (1968).

[3] Hatc, C. A., and T. A. Porsching: Computing the maximal eigenvalue and eigenvector of a nonnegative irreducible matrix. SIAM J. Num. Anal. 5, $470-474$ (1968).

\section{Ludwig Elsner}

Institut für Angewandte Mathematilk I der Universität Erlangen.

Erwin-Rommel-Straße 60, D-852 Erlangen

Deutschland 